\title{
Advanced Glycation Endproducts Promote Adhesion Molecule (VCAM-1, ICAM-1) Expression and Atheroma Formation in Normal Rabbits
}

\author{
Helen Vlassara,* Hubert Fuh, * Thomas Donnelly, \\ and Myron Cybulsky ${ }^{\dagger}$ \\ *The Picower Institute for Medical Research, Manhasset, \\ New York, U.S.A. \\ ${ }^{\dagger}$ Department of Pathology, Brigham \& Women's Hospital, Boston, \\ Massachusetts, U.S.A.
}

\begin{abstract}
Background: Reactive glucose-protein intermediates and advanced glycation endproducts (AGEs) are shown to colocalize with atheromatous lesions and to trigger complex chemical and biological responses through interaction with vessel wall elements. In diabetes and renal insufficiency, atherosclerosis is common, as are elevated levels of serum and vascular tissue AGEs. In the present study, AGEs supplied exogenously to normal animals elicited vascular and renal pathology.

Materials and Methods: Nondiabetic rabbits were injected intravenously with low doses of AGE-modified rabbit serum albumin (AGE-RSA, $16 \mathrm{mg} / \mathrm{kg} /$ day) for 4 months alone, or combined with a brief terminal period ( 2 weeks) of a cholesterol-rich diet (CRD) ( $2 \%$ cholesterol, $10 \%$ corn oil). AGE-RSA associated expression of vascular cell adhesion molecules and the development of atheromatous changes within the aorta were determined by immunohistology.

Results: The AGE content of aortic tissue increased by 2.2-fold in AGE-treated and by 3.2-fold in AGE + CRDtreated rabbits compared with normal saline-treated
\end{abstract}

control rabbits $(p<0.025$ and 0.001 , respectively). Serum AGE levels in AGE groups rose up to 3-fold above the controls $(p<0.025$ and $p<0.01)$. Ascending aortic sections from AGE-treated rabbits showed significant focal intimal proliferation, enhanced endothelial cell adhesion with infrequent intimal macrophages, oil-red-O staining lipid deposits and positive focal expression of vascular cell adhesion molecule-1 (VCAM-1), and intercellular adhesion molecule-1 (ICAM-1), a pattern not observed in controls. These AGE-induced changes were markedly enhanced in animals cotreated with AGEs and a brief period of CRD. Lesions consisted of multifocal atheromas, containing foam cells, massive lipid droplets, and strong endothelial expression of VCAM-1 and ICAM-1 restricted to the affected areas.

Conclusions: This study provides in vivo evidence for a causal relationship between chronic AGE accumulation and atherosclerosis independent of diabetic hyperglycemia, and suggests the utility of this animal model for the study of diabetic vascular disease in relation to glycation.

\section{INTRODUCTION}

Among patients with diabetes mellitus, cardiovascular disease is highly prevalent $(1-3)$. This has been attributed to an acceleration of atherosclerotic processes that more extensively culminate in the development of vascular lesions

Address correspondence and reprint requests to: Helen Vlassara, The Picower Institute for Medical Research, 350 Community Drive, Manhasset, NY 11030, U.S.A. earlier than normal. Many pathophysiological changes contribute to the typical atherosclerotic progression and include increased vascular permeability, enhanced procoagulant activity, and impaired vasodilation. Alterations in adhesive properties of the endothelial surface, focal adherence, and subsequent migration of mononuclear cells into the subendothelial space, together with myointimal proliferation and accumulation of serum lipoproteins, soon lead to widening of in- 
timal and medial layers and additional accretion of cellular and noncellular material, as the complex process of intraluminal plaque formation continues $(4,5)$. Initial monocyte recruitment is thought to be due to induction of endothelialleukocyte adhesion molecules, such as intercellular adhesion molecule-1 (ICAM-1) and vascular cell adhesion molecule-1 (VCAM-1), which coincide with the earliest phases of local endothelial activation and contribute to endothelial-monocyte interactions in early atherogenesis $(6,7)$.

Recent clinical evidence indicates that reduction of mean blood glucose level can significantly lower the risk for development of atherosclerosis and vascular disease, as well as other complications of diabetes (8). These large scale studies support strongly the belief that, among other risk factors, sustained hyperglycemia is a prerequisite for the development of vascular complications and other sequelae of diabetes. Advanced glycation endproducts (AGEs), the late reaction products of the nonenzymatic pathways causing proteins, lipids, and nucleic acids to become spontaneously modified by glucose, accumulate in diabetes as a direct consequence of prolonged hyperglycemia. In particular, AGE-modified proteins and lipids have been shown to accumulate in vascular tissues as a function of increasing age and of diabetes $(9,10)$, and to colocalize with atheromatous lesions (11). Patients with end-stage renal disease (ESRD), a group that suffers excessive morbidity and mortality due to accelerated atherosclerosis (12) in addition to their underlying nephropathy, also exhibit exaggeratedly elevated serum AGEs (13). These circulating AGE-bearing molecules include a low molecular weight class, known as AGE-peptides, that are chemically reactive and tend to attach covalently to vessel wall and plasma components (14). Numerous in vitro studies have suggested that, through interactions with cell surface AGE-specific receptors, AGEs induce vascular tissue alterations, such as increased endothelial cell permeability (15), adhesion molecule expression (16), mononuclear cell migration (17), procoagulant activity (15), nitric oxide inactivation (18), oxygen reactive intermediates (19), and other factors which could predispose diabetic tissues to vascular dysfunction earlier than in nondiabetic individuals.

The pathogenic links between hyperglycemia, AGE accumulation, and characteristic tissue pathology have been tested in several animal models, showing that the short-term administra- tion of exogenous AGEs to nondiabetic rats, rabbits, or mice results in systemic vascular dysfunction (20), can significantly enhance the size of experimentally induced cerebral strokes (Zimmerman et al., submitted), and can lead to early (21) and advanced end-organ damage (e.g., glomerular sclerosis) (22). However, the long-term in vivo consequences of cumulative AGE deposition on the structure and function of the aortic wall, assessed for instance by adhesion molecule expression and atheroma formation independent of hyperglycemia, has not been determined.

In this report, we present evidence indicating that in vivo exposure of euglycemic rabbit aortic vessel wall tissues to AGE-modified homologous serum albumin can induce significant local biochemical and physiological alterations, including enhanced adhesion molecule (VCAM-1, ICAM-1) expression, such that the evolution of frank atheromatous lesions is markedly accelerated.

\section{MATERIALS AND METHODS}

\section{Preparation of Advanced Glycation Products}

Rabbit serum albumin (RSA) (Sigma Chemical Co., St. Louis, MO, U.S.A.) was passed over an Affi-Gel Blue column (Biorad Lab., Richmond, CA, U.S.A.), a heparin-Sepharose CL-6B column (Pharmacia, Piscataway, NJ, U.S.A.) and an endotoxin-binding affinity column (Detoxigel, Pierce, IL, U.S.A.) to remove possible contaminants (19-21 and Zimmerman et al., submitted). RSA modified by advanced glycation endproducts was prepared as described (19-21 and Zimmerman et al. submitted). AGE levels were measured by AGE-specific ELISA based on polyclonal anti-RNAse (23) or on monoclonal anti-AGE IgG (provided by Dr. H. Founds, Alteon, Inc.). (AGErat albumin: $92 \mathrm{AGE} \mathrm{U/mg} \mathrm{protein,} \mathrm{unmodified}$ rat albumin control: $0.8 \mathrm{U} / \mathrm{mg}$ ). Each reagent contained $<0.2 \mathrm{ng} / \mathrm{ml}$ of endotoxin (E-Toxate, Sigma).

\section{Animal Studies}

Male New Zealand White (NZW) (Pasteurellafree) rabbits ( $n=20,2.5 \mathrm{~kg}$ each) obtained from Hare-Marland (Hewitt, NJ, U.S.A.) were used in these studies, which were conducted in accordance with the Picower Institute Laboratory Animal Center guidelines. Rabbits were individually housed and after a 2-week adaptation period, 
randomly assigned to four groups ( $n=5$ /group) and fed commercially available pelleted diet (48 $\mathrm{g} / \mathrm{kg} /$ day) (Lab-Rabbit Chow \#8526, Ralston-Purina, NC, U.S.A.). Two of the groups were given daily injections of AGE-modified rabbit serum albumin (AGE-RSA) $(16 \mathrm{mg} / \mathrm{kg} /$ day) through the marginal ear vein for four months in normal saline. Two groups were treated with normal saline (controls). During the last 2 weeks of treatment, one of the AGE-RSA-treated groups and a normal control group were placed on a cholesterol-rich diet ( $2 \%$ USP cholesterol, $10 \%$ corn oil); the remaining animals consumed an isocaloric control diet which contained $12 \%$ corn oil without cholesterol supplementation. Food intake was recorded daily and body weights were monitored weekly. Blood samples were collected once a month and at the end of the treatment period for determination of serum AGE (23), cholesterol and triglyceride levels. Plasma cholesterol was measured enzymatically using a Roche Cobas Mira random-access analyzer, while total triglycerides were measured by a Roche Cobas Fara II centrifugal analyzer. At the end of the treatment period the animals were killed by overdose of pentobarbital $(100 \mathrm{mg} / \mathrm{kg}$, Nembutal, Abbott Lab., IL, U.S.A.) The aorta was flushed through the left ventricle with approximately 150-200 $\mathrm{ml}$ of chilled phosphate buffered saline, and was dissected into four segments: aortic arch, ascending thoracic, descending thoracic, and abdominal aorta. Each standardized segment was further divided into subsections for immunohistochemistry, histopathology, and AGE analysis. Standardized $1 / 3$ organ samples from heart, liver, and kidneys were also taken for similar evaluation. Specimens for immunohistochemistry or AGE measurements were snap-frozen in liquid nitrogen-chilled isopentane, transferred to liquid nitrogen, and stored at $-80^{\circ} \mathrm{C}$.

To determine AGE accumulation within the aorta, thoracic aorta segments were finely minced, delipidated with acetone/chloroform (1:1), and digested with $1 / 100(\mathrm{w} / \mathrm{w})$ Type VII collagenase (Sigma) for $48 \mathrm{hr}$ at $37^{\circ} \mathrm{C}$ for determination of collagen content as described (24). Tissue AGE levels were determined by ELISA on protein fractions solubilized by collagenase treatment as described (23).

\section{Histology}

Tissue samples $\left(3-\mathrm{mm}^{3}\right)$ were fixed in cold $10 \%$ buffered formalin (Millonig's Modified Buffered
Formalin, $\mathrm{pH}$ 7.4) for $24 \mathrm{hr}$ and embedded in paraffin for sectioning. Paraffin sections from all animal groups ( $n=5$ /group) were processed in triplicate by staining with hematoxylin and eosin, and Verhoeff-Van-Gieson for routine histological examination in a blinded fashion. Serial 5-6 $\mu \mathrm{m}$-thick frozen aortic tissue sections were fixed in acetone at $-20^{\circ} \mathrm{C}$ for $5^{\prime}$ and incubated with anti-VCAM-1 mab Rbl/9, anti-ICAM-1 mab Rb2/3, and anti-E-selectin (ELAM-1) mab (mouse IgGl hybridoma supernatants), all previously characterized and shown to recognize the corresponding rabbit cell adhesion molecules $(7,16)$. Species-appropriate biotinylated secondary antibodies (horse anti-mouse IgG) were applied, followed by avidin-biotin peroxidase complexes (ABC Elite kit; Vector Labs, Burlingame, CA, U.S.A.). Additional frozen sections were stained with oil-red-O for evaluation of lipid content.

\section{Data Analysis}

Serum and tissue AGE content, as well as plasma cholesterol and triglyceride values were analyzed by a one-way analysis of variance (significance level $p<0.05$ ). Histological evaluation of aortic tissue sections involved examination of at least three serial cross-sections obtained at the identical level of the proximal ascending, descending and abdominal aorta, and was performed in a blinded fashion by three independent investigators for the presence and extent of atherosclerotic lesions. Immunohistochemical sections were also scored as positive or negative for each adhesion molecule.

\section{RESULTS}

Prior studies of the effects of chronic daily treatment of normal rabbits with AGE-modified or native rabbit serum albumin $(100 \mathrm{mg} / \mathrm{kg} / \mathrm{day}$, i.v. $\times 4$ weeks) indicated that pathology was absent in species-specific albumin-treated controls or in animals cotreated with AGE-RSA and the advanced glycation inhibitor, aminoguanidine (Refs. 20 and 21, and Zimmerman et al., submitted). Thus, in the present study, to explore the cumulative synergistic impact of hyperlipidemia, experimental groups included rabbits which were exposed to a brief period of cholesterol-rich diet (CRD) alone or CRD was added to AGE treatment (AGE + CRD). During the 4-month period of daily intravenous infusions of 
TABLE 1. Rabbit plasma cholesterol levels during administration of AGE-RSA ${ }^{a}$ and CRD $^{b}$

\begin{tabular}{llrr}
\hline Group Treatment & Baseline & 14 Weeks & 16 Weeks \\
\hline Control (normal saline) & $56 \pm 22$ & $67 \pm 12$ & $68 \pm 24$ \\
AGE-RSA & $62 \pm 28$ & $87 \pm 22$ & $78 \pm 16$ \\
AGE-RSA + CRD & $73 \pm 16$ & $53 \pm 18$ & $1380 \pm 322^{c}$ \\
CRD $^{b}$ & $50 \pm 2$ & $72 \pm 30$ & $966 \pm 238^{c}$ \\
\hline
\end{tabular}

Data are expressed in $\mathrm{mg} / \mathrm{dl}$ (mean $\pm \mathrm{SD}, n=5 /$ group).

${ }^{a}$ AGE-RSA was infused at $16 \mathrm{mg} / \mathrm{kg} /$ day i.v. for 16 weeks.

${ }^{b} \mathrm{CRD}$, cholesterol-rich diet ( $2 \%$ USP cholesterol $+10 \%$ corn-oil) was introduced at last 2 weeks of study.

${ }^{c}$ Statistically different from control $(p<0.001)$ and from AGE-RSA group $(p<0.002)$.

AGE-modified albumin (or normal saline) food intake and growth rates remained stable among all groups. Starting on the 14th week of AGERSA treatment, consumption of the atherogenic diet (CRD) by two groups, one AGE-treated, the other saline, induced significant elevations in plasma cholesterol levels within 5 days that lasted for the remaining 8-10 days of the study (Table 1). During this brief exposure to highcholesterol diet, plasma ALT and AST transaminases remained stable, reflecting no evidence of liver damage which is often associated with much longer maintenance on atherogenic diets (data not shown).

The daily infusion of rabbits with low amounts of AGE-RSA over a period of 4 months resulted in aortic tissue AGE accumulation (2.2fold increase), that was more marked when AGE-treated animals were placed on CRD (AGE + CRD; 3.2-fold increase), compared with aortas from rabbits treated with CRD alone $(p<0.025$, $p<0.001$, respectively) or control diet rabbits ( $p<0.025, p<0.01$, respectively) (Fig. 1A). Similarly circulating AGE-protein levels in AGERSA and AGE + CRD rabbits rose up to 3-fold above controls $(p<0.005, p<0.025$, respectively) (Fig. 1B).

\section{Microscopic Evaluation}

Aortic arch, ascending and descending portions of the thoracic aorta contained more advanced lesions than abdominal sections of the aorta. Accordingly, identical sites in the ascending aortas were chosen from each group for immunohistochemistry. The aortic intimas of four out of five AGE-treated rabbits exhibited mild focal intimal proliferation and thickening, with infrequent in- timal macrophages (Fig. 2a). Examination of multiple adjacent sections revealed leukocytes adhering to the endothelial monolayer (Fig. 2a). Some sections revealed moderate focal medial degeneration. Oil-red-O staining revealed modest amounts of diffuse subintimal fatty deposition (Fig. 2e). Although mild in severity, the histological features of these aortic sections after four
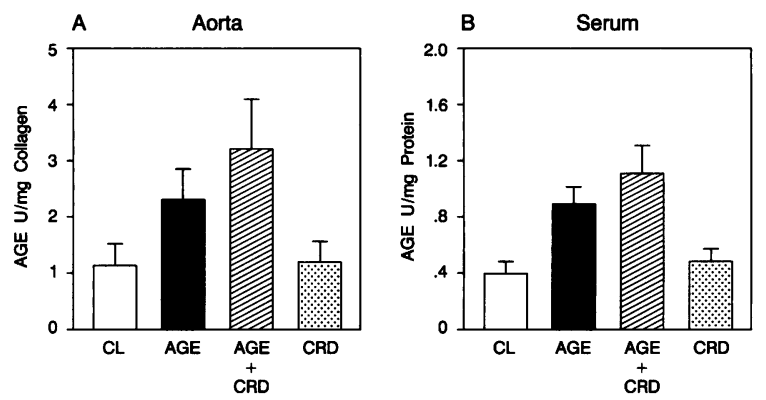

FIG. 1. Rabbit aortic tissue (A) and serum (B) AGE levels after intravenous administration of AGE-modified rabbit serum albumin (AGE-RSA)

Following treatment with either AGE-RSA (16 mg/ $\mathrm{kg} /$ day, i.v.) (AGE) alone for 4 months, 2\% USP cholesterol-rich diet (CRD) supplemented with $10 \%$ corn oil for 2 weeks, normal saline control $(0.5 \mathrm{ml} /$ day, i.v.) (CL), or AGE for 4 months plus CRD for last 2 weeks (AGE + CRD), AGE levels were determined in collagenase-digested aortic tissue extracts using an AGE-specific ELISA. (A) Aorta AGE levels (mean $\pm \mathrm{SEM}, n=5$ /group). Significant $p$ values: $A G E$ versus $C L,<0.025, A G E$ versus $C R D<0.025$; $\mathrm{AGE}+\mathrm{CRD}$ versus $\mathrm{CL}<0.01, \mathrm{AGE}+\mathrm{CRD}$ versus CRD $<0.001$. (B) Serum AGE levels (mean \pm SEM, $n=5$ /group) $p$ values: AGE versus $C L$ and $C R D<$ 0.005 each; $\mathrm{AGE}+\mathrm{CRD}$ versus $\mathrm{CL}$ and $\mathrm{CRD}<$ 0.025 each. 

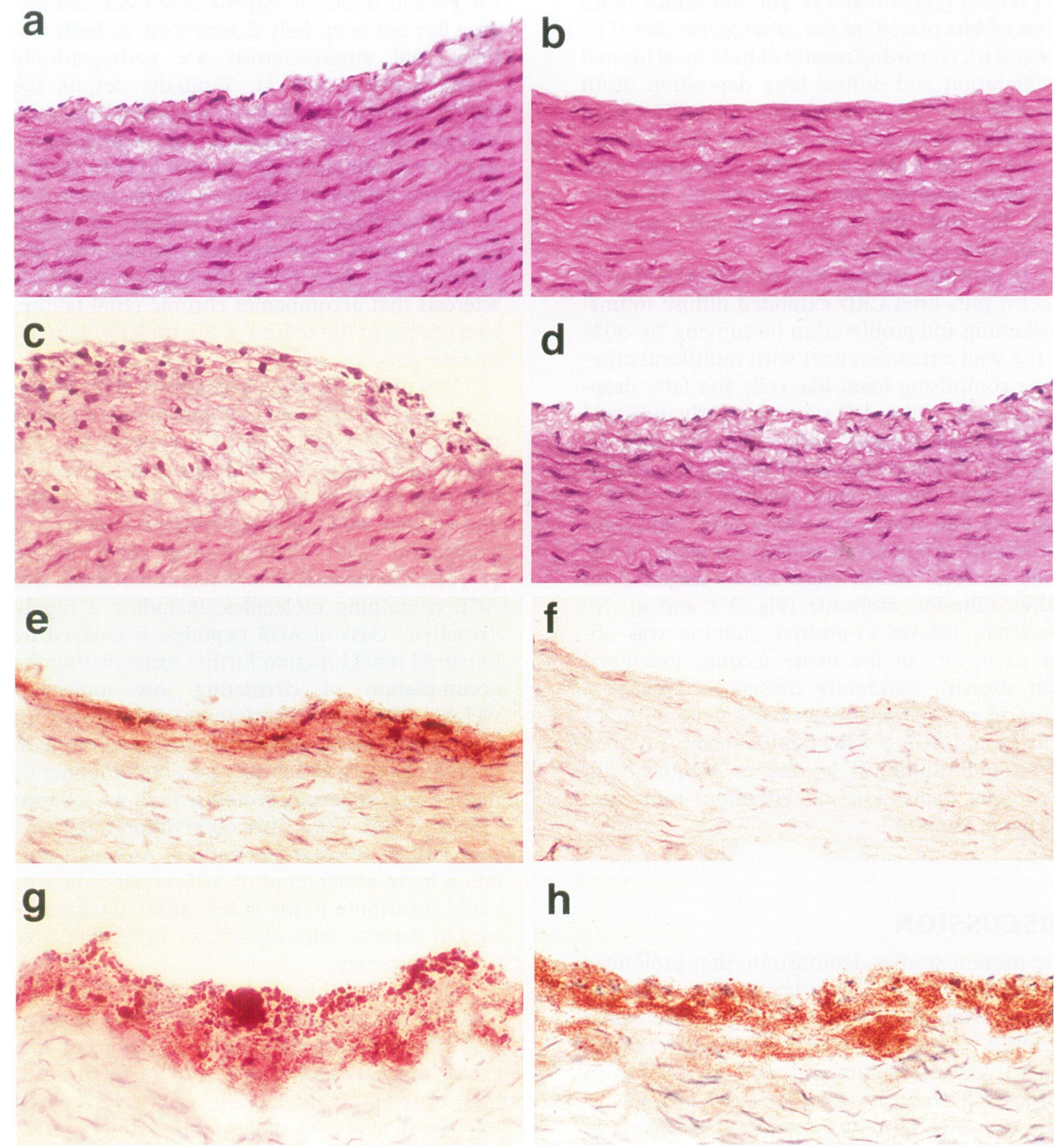

\section{h}

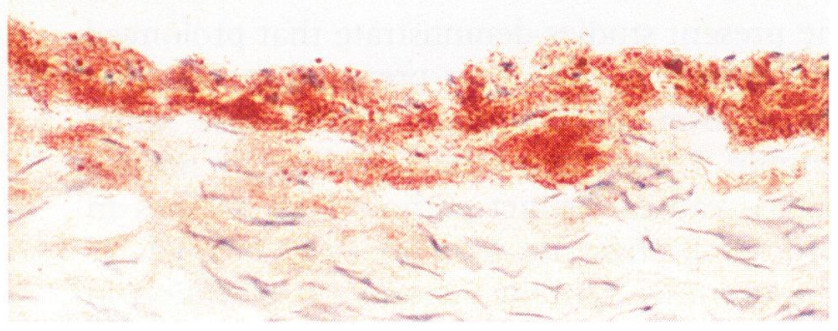

FIG. 2. Light microscopy micrographs showing eosin/hematoxylin (a-d) and oil-red-o (e-h) staining of aortic sections from euglycemic rabbits treated with AGE-modified rabbit serum albumin (AGERSA) or saline with varying diet.

Treatments of rabbits were as follows: (a,e) AGE-RSA, $16 \mathrm{mg} / \mathrm{kg} / \mathrm{d}$ i.v. $\times 4$ months; (b,f) normal saline, $0.5 \mathrm{ml} /$ day, i.v. $\times 4$ months; (c,g) AGE-RSA, $16 \mathrm{mg} / \mathrm{Kg} / \mathrm{d}$ i.v. $\times 4$ months plus $2 \%$ cholesterol-rich diet $(\mathrm{CRD}) \times 2$ weeks $(\mathrm{AGE}+\mathrm{CRD}) ;(\mathrm{d}, \mathrm{h}) \mathrm{CRD}$ alone $\times 2$ weeks. $(\mathrm{HGE} ; 200 \times$.) 
months of exposure to AGEs was significantly different from the sections of saline-treated control rabbits (Fig. $2 \mathrm{~b}$ and f), and not unlike those from rabbits placed on the atherogenic diet (Fig. $2 \mathrm{~d}$ and $\mathrm{h}$ ), consisting mostly of mild focal intimal proliferation and diffuse fatty deposition. Both the AGE and CRD groups exhibited positive focal expression of endothelial VCAM-1 (Fig. 3 a and d) and ICAM-1 (Fig. 3 e and h), compared with untreated controls that contained no VCAM-1 (Fig. 3b) and only occasional ICAM-1-positive endothelial cells (Fig. 3f).

In contrast, animals treated with AGE (for 16 weeks) plus brief CRD exhibited diffuse intimal thickening and proliferation (occupying 70-90\% of the wall circumference) with multifocal atheroma comprising foam-like cells and fatty droplets (Fig. 2c). Oil-red-O staining clearly indicated that the lipid deposits were largely intracellular (Fig. 2g). The endothelium overlying these complex lesions was strongly positive for VCAM-1 (Fig. 3c) and ICAM-1 staining (Fig. 3g), compared with uninvolved adjacent regions of the same sections, which were mostly negative for either adhesion molecule (Fig. $3 \mathrm{c}$ and g). No E-selectin (ELAM-1)-positive staining was observed in any of the tissue sections examined (not shown). Randomly chosen sections from thoracic and abdominal aorta segments of AGEinfused and AGE + CRD treated rabbits revealed occasional multifocal lesions or plaques with moderate expression of adhesion molecules (data not shown).

\section{DISCUSSION}

The present studies demonstrate that prolonged administration of in vitro prepared species-specific AGE-modified protein, in this case rabbit albumin infused into otherwise healthy normal rabbits, causes distinct pathological changes in aortic wall structure akin to early changes of atherogenesis. Moreover, transient elevation in plasma lipid concentration induced by dietary changes synergized potently with AGE administration and induced rapidly evolving atheromatous lesions. Taken together with previous evidence implicating AGEs in a number of biochemical, cellular, and early vascular pathophysiological events in vivo, these new data further support a cause-and-effect relationship between chronic AGE accumulation and atherosclerosis.

Although high levels of AGE immunoreactivity has been observed within the atheroscle- rotic plaque of diabetic vessels (11), the specific in vivo role of late glycation products (AGEs) in the establishment of diabetic macrovascular disease has not been fully determined, as both diabetes and atherosclerosis are pathogenically complex diseases (1-3). Similarly, despite the strong association of AGE accumulation with normal aging, the pathogenic role of progressive AGE accumulation in the gradual thickening and increased rigidity of large vessel walls, referred to as arteriosclerosis, with or without attendant atherosclerosis, is not well understood. Even less understood is the marked acceleration of atherosclerosis that accompanies chronic renal failure, irrespective of the nature of the underlying renal disease (25).

Vascular tissue and plasma levels of AGEs are reported to be elevated in connection with renal insufficiency (13). The AGE content of the low molecular weight $(<10 \mathrm{kD})$ serum fraction that may represent in part the degradation of tissue and cellular AGEs, is independently elevated in association with diabetes and renal failure $(13,14)$. The normal renal excretion of small size AGE containing molecules, including a highly "reactive" class of AGE peptides, is delayed by impaired renal function further exacerbating the accumulation of circulating low molecular weight AGEs. In experimental incubations in vitro, a large portion of these reactive serum AGE peptides readily attach to vascular components, matrix and/or serum proteins, such as collagen (14) and LDL (26). This observation suggested that similar "second generation" AGE accumulation by re-attachment of AGE peptides in vivo could contribute to the acceleration of tissue injury in patients with diabetic or nondiabetic renal insufficiency.

This potential for in vivo vascular "toxicity" of AGEs independent of hyperglycemia or aging was first demonstrated directly in groups of otherwise normal rats and rabbits treated daily with high doses $(100 \mathrm{mg} / \mathrm{kg} /$ day $)$ of species-specific AGE-modified serum albumin over study periods of 60 days. This chronic exposure resulted in increased levels of covalently attached vascular tissue AGEs and was associated with widespread vascular leakage, mononuclear infiltration, and vasodilatory impairment, all of which were prevented by the coadministration of aminoguanidine, an AGE-crosslink inhibitor (20). Similar studies in SJL mice over a shorter treatment period demonstrated a rapid upregulation of specific genes for growth factor and extracellular matrix components in the kidney, in association 

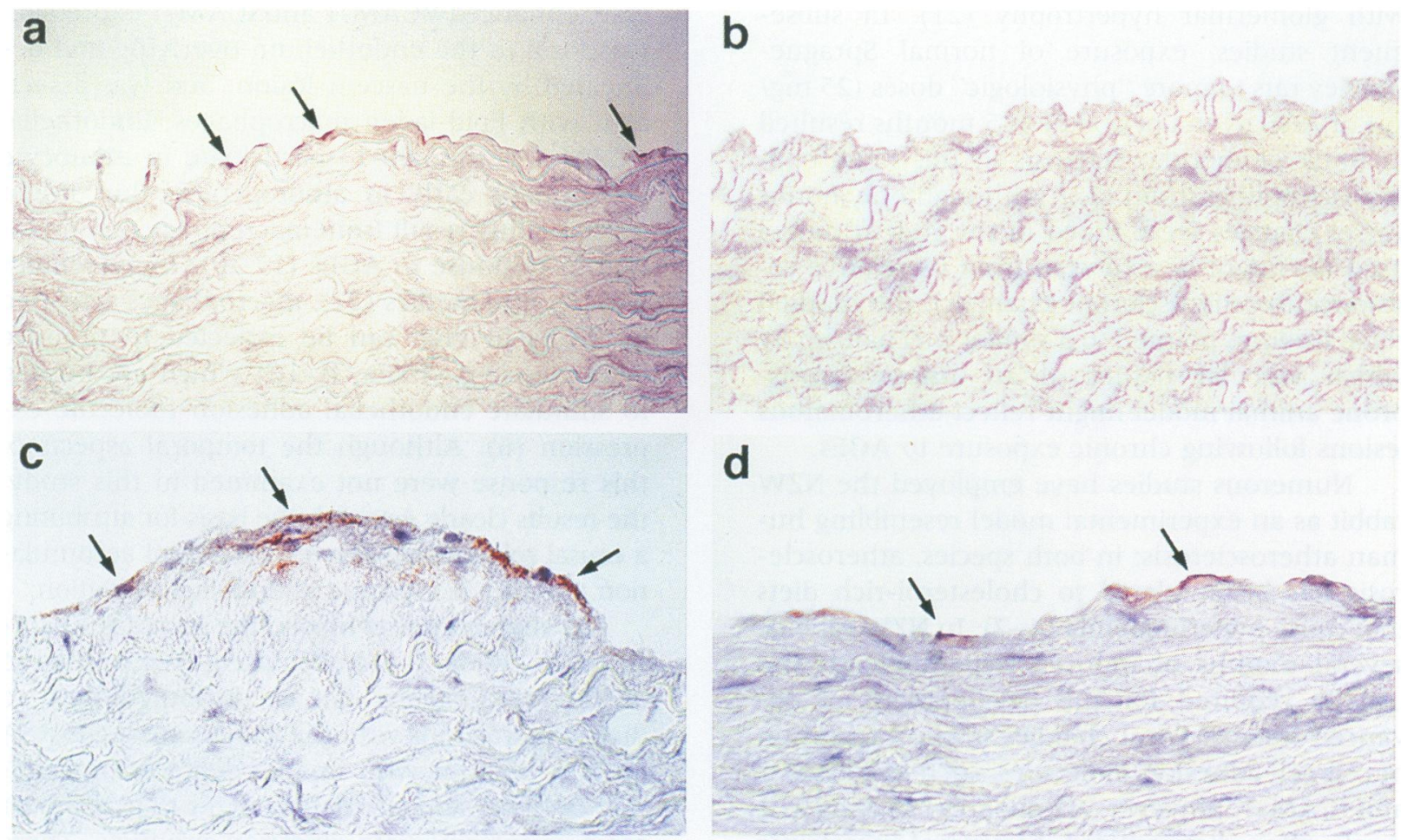

e

f
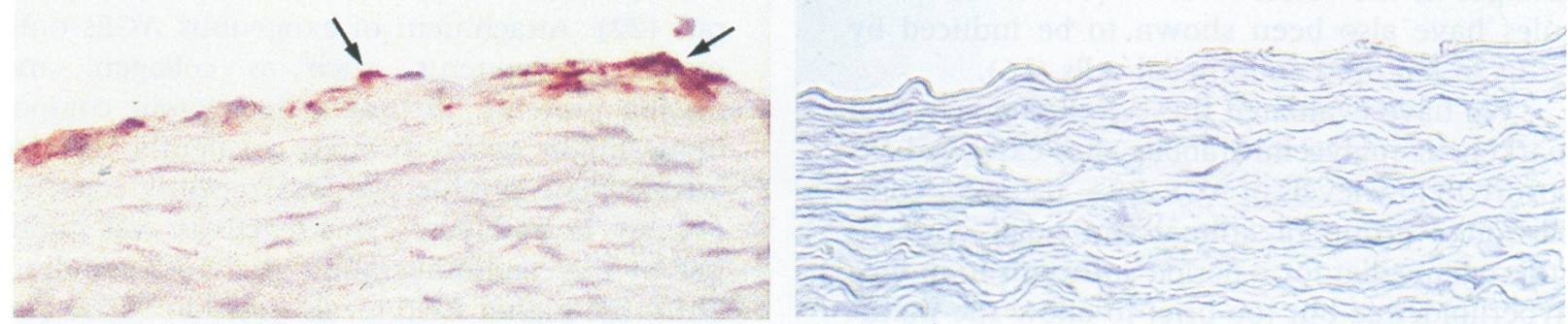

g

$\mathbf{h}$
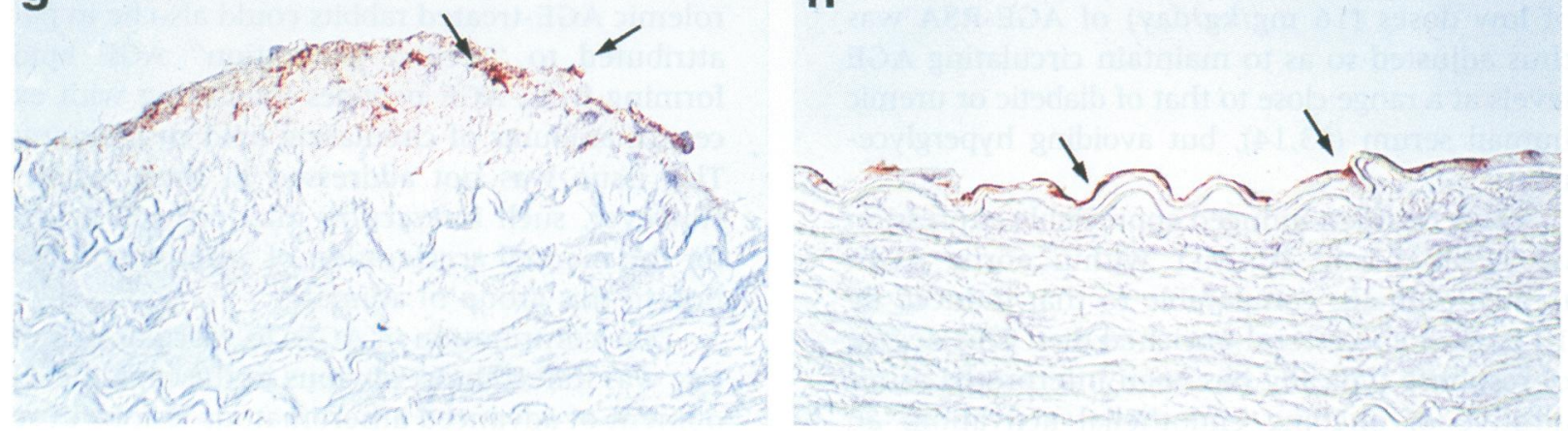

FIG. 3. Immunohistochemical staining of endothelial vascular adhesion molecule-1 (VCAM-1) (a-d), and intercellular adhesion molecule-1 (ICAM-1) expression in aortic sections of euglycemic rabbits treated with AGE-modified rabbit serum albumin (AGE-RSA) or saline with varying diet

Treatments for rabbits were as follows: (a,e) AGE-RSA, $16 \mathrm{mg} / \mathrm{kg} / \mathrm{d}$ i.v. $\times 4$ months; (b,f) normal saline, $0.5 \mathrm{ml} \times$ 4 months; (c,g) AGE-RSA, $16 \mathrm{mg} / \mathrm{Kg} / \mathrm{d}$ i.v. $\times 4$ months, plus cholesterol-rich diet (CRD) $\times 2$ weeks $(\mathrm{AGE}+$ $\mathrm{CRD})$; $(\mathrm{d}, \mathrm{h}) \mathrm{CRD}$ alone $\times 2$ weeks. Arrowheads indicate areas of respective endothelial adhesion molecule expression. 
with glomerular hypertrophy (21). In subsequent studies, exposure of normal SpragueDawley rats to more "physiologic" doses $(25 \mathrm{mg} /$ $\mathrm{kg}$ ) of AGE over a period of 4-5 months resulted in frank glomerular sclerosis in this nephropathy-susceptible strain (22). Although only minor vessel changes were noted in the rodent model used (as might be expected from its relative resistance to atherosclerotic changes), the marked renal findings pointed to a similar mechanism by which vascular tissues of an atherosclerosisprone animal model might reflect atheromatous lesions following chronic exposure to AGEs.

Numerous studies have employed the NZW rabbit as an experimental model resembling human atherosclerosis; in both species, atherosclerosis has been related to cholesterol-rich diets that cause hyperlipidemia (4-7). In NZW rabbits, several months of induced hyperlipidemia are usually required for the development of advanced lesions. Focal changes in the expression of cell/cell adhesion molecules, such as VCAM-1 and ICAM-1, however, are apparent as early as 1 week after diet-induced hypercholesterolemia (6). Induction of these genes has thus been taken as a useful marker of endothelial surface activation and as an early indication of atherosclerotic changes in the vessel wall. Both of these molecules have also been shown to be induced by AGEs in cultured endothelial cells (16).

We have evaluated these well-characterized markers in euglycemic rabbits after extended exposure to low levels of AGE-RSA, using for comparison a group of animals provided with an atherogenic diet for a period sufficient to induce hyperlipidemia but too brief to allow the maturation of frank lesions. The daily administration of low doses (16 mg/kg/day) of AGE-RSA was thus adjusted so as to maintain circulating AGE levels at a range close to that of diabetic or uremic human serum $(13,14)$, but avoiding hyperglycemia.

This regimen induced appreciable expression of VCAM-1 and ICAM-1 within aortic AGEtreated animals, comparable to that induced by 10 days of cholesterol-enriched diet. This degree of response typically has been interpreted as indicative of ongoing endothelial activation, an early feature of arterial lesion formation. At this stage, in both groups, modest amounts of diffuse lipid deposition (by oil-red-O) and infrequent adherent leukocytes were evident. Interestingly, more advanced atherosclerotic lesions were induced when the same AGE treatment was combined with short-term hyperlipidemia. In this case, enhanced VCAM-1 and ICAM-1 expression was focal in the endothelium overlying and delineated by the nascent lesion, and was associated with lipid-laden macrophages. Endothelial VCAM-1 is thought to participate in monocyte recruitment early in atherogenesis, but might subsequently result from macrophage accumulation in response to AGEs $(17,20)$. In agreement with in vitro studies (27), macrophages activated by AGEs in vivo can be expected to produce cytokines (e.g., TNF $\alpha$, IL- $1 \beta$ ) which are known to stimulate endothelial adhesion molecule expression (6). Although the temporal aspects of this response were not examined in this study, the results clearly establish the basis for attributing a causal relationship between the local accumulation of AGEs in vivo and endothelial activation.

As suggested previously, the increase in aortic tissue resident and circulating AGEs noted in AGE-treated rabbits can be attributed both to direct interactions with the exogenous AGE-RSA and to reactions with smaller degradation products of tissue and circulating AGEs that also bear reactive intermediate glycation products. These smaller fragments are largely cleared via the kidney as AGE-rich peptide fragments; a previously marked increase in AGE-immunoreactive material was noted in the urine of AGE-RSA treated rats (22). Attachment of exogenous AGEs onto matrix components, such as collagen, and plasma proteins, including lipoprotein components might occur through covalently reactive intermediate products as was recently reported $(14,26)$. In both cases, this reactivity was inhibited by the coadministration of aminoguanidine. Thus, the higher AGE levels noted in aortas and in circulation of transiently hypercholesterolemic AGE-treated rabbits could also be in part attributed to "second generation" AGE lipids forming from AGE peptides interacting with excessive amounts of circulating lipid components. This issue was not addressed in these studies. However, such interaction may provide a basis for the marked acceleration of atheroma formation in this group of animals.

The contribution of AGEs to vascular pathology was indeed most obvious in the rapid development of advanced atheromatous lesions in response to brief hyperlipidemia; consistent with earlier studies, such lesions were not generated by 2 weeks of dietary manipulation alone (6). The additive effects of AGE-RSA and high-cholesterol diet observed in these studies might reflect either increased LDL cholesterol trapping within tissues due to the accumulation of locally 
deposited reactive AGEs, or by inhibition of normal lipid clearance mechanisms due to formation of AGE lipid particles (26). The latter is plausible, based on the recent identification of increased levels of an AGE-immunoreactive form of human plasma LDL cholesterol found in patients with diabetes or renal insufficiency, both of which had high circulating AGE levels (26). Whether in tissues or in circulation, AGE lipid formation is followed by the generation of oxidatively modified lipid (26) a powerful atherogenic substance (28).

The spectrum of cell activation and pro-atheroma changes observed in these chronically AGE-treated animals may be mediated in part by the induction of cytokines (TNF $\alpha, \mathrm{IL}-1 \beta$, IL-6) and growth factors (IGF-IA, PDGF, and TGF $\beta 1$ ), which can regulate both cellular proliferation and protein synthesis. Each of these, has been implicated in the complex network of interactions controlling growth and matrix production in human and experimental systems $(4,5)$. The cellular mechanisms underlying the effects presented here, including oxidant stress (19) and altered cellular properties (15-17), are still unclear, however, the contribution of AGE-specific receptors present on endothelium, macrophages, $\mathrm{T}$ cells, and smooth muscle cells, all major players in atherogenesis, is highly probable (10); in culture these cells are shown to respond with altered expression of a similar spectrum of mediator molecules via surface AGE-binding sites (10,15-17). The present data, however, do not address these mechanistic possibilities. Furthermore, under the conditions used, animals exposed to native rabbit serum albumin presented no serologic or light microscopic changes attributable to a systemic immune response. This reduces the likelihood of an immune complex-like mechanism underlying the formation of AGERSA generated lesions.

The pattern of aortic changes presented here recapitulate many elements in the progression of human atherosclerosis, irrespective of underlying cause (e.g., hyperlipidemia, diabetes, aging). Although there can be no central role for hyperglycemia in aging-related vascular wall injury, given the gradual accumulation of AGEs in aging tissues $(9,10)$, it is tempting to speculate that the chronically deposited AGE substances may generate a vulnerable substrate in susceptible individuals over time. Although further studies are necessary to establish this association, the model presented in these studies may prove useful in the study of both diabetes and aging-associated large vessel disease.

The work presented here extends the in vivo evidence that AGEs play an independent role in inducing vascular disease in otherwise normal aortas. Thus, the presence of excessive AGEs in tissues or the circulation may critically affect the progression of vascular damage in patients with diabetes or renal insufficiency.

\section{ACKNOWLEDGMENTS}

We would like to thank Dr. Kirk Manogue for his valuable guidance and to Ms. Donna Bove for her editorial assistance. These studies were supported in part by The National Institutes of Health Grants AGO-6943 and AGO-9453 (to HV) and HL 45563 (MC).

\section{REFERENCES}

1. Ruderman NB, Haudenschild C. (1984) Diabetes as an atherogenic factor. Progr. Cardiovasc. Dis. 26(5): 373-412.

2. Manske CL, Wilson RF, Wang Y, Thomas W. (1992) Prevalence of, and risk factors for, angiographically determined coronary artery disease in type I diabetic patients with nephropathy. Arch. Intern. Med. 152: 2450-2455.

3. Krolewski AS, Kosinski EJ, Warram JH, et al. (1987) Magnitude and determinants of coronary artery disease in juvenile-onset, insulin-dependent diabetes mellitus. Am. J. Cardiol. 59: 750-755.

4. Ross R. (1986) The pathogenesis of atherosclerosis-an update. N. Engl. J. Med. 314: 488-500.

5. Munro JM, Cotran RS. (1988) The pathogenesis of atherosclerosis and inflammation. Lab. Invest. 58: 249-261.

6. Li H, Cybulsky MI, Gimbrone Jr MA, Libby P. (1993) An atherogenic diet rapidly induces VCAM-1, a cytokine-regulatable mononuclear leukocyte adhesion molecule, in rabbit aortic endothelium. Arteriosclerosis and Thrombosis 13: 197-204.

7. Cybulsky MI, Gimbrone Jr MA. (1991) Endothelial expression of a mononuclear leukocyte adhesion molecule during atherogenesis. Science 251: 788-791.

8. The Diabetes Control and Complications Trial Research Group. (1993) The effect of intensive treatment of diabetes on the development and progression of long-term complications in insulin-dependent diabetes mellitus. N. Engl. J. Med. 329: 977-986. 
9. Monnier VM, Cerami A. (1981) Nonenzymatic browning in vivo: possible process foraging of long-lived proteins. Science 211:491493.

10. Vlassara H, Bucala R, Striker L. (1993) Pathogenic effects of advanced glycosylation; Biochemical, biological, and clinical implications for diabetes and aging. J. Lab. Invest. 70(2): $138-151$.

11. Nakamura Y, Horii Y, Nishino T, et al. (1993) Immunohistochemical localization of advanced glycosylation endproducts (AGEs) in coronary atheroma and cardiac tissue in diabetes mellitus. Am. J. Pathol. 143(6): 16491656.

12. National Institute of Diabetes and Digestive and Kidney Diseases. (1992) Renal Data System Annual Data Report. National Institutes of Health, Bethesda.

13. Makita Z, Radoff S, Rayfield EJ, et al. (1991) Advanced glycosylation endproducts in patients with diabetic nephropathy. $N$. Engl. $J$. Med. 325: 836-842.

14. Makita Z, Bucala R, Rayfield EJ, et al. (1994) Reactive glycosylation endproducts in diabetic uraemia $\delta$ treatment of renal failure. Lancet 343: 1519-1522.

15. Esposito C, Stern D, Gerlach H, Vlassara H. (1989) Endothelial receptor-mediated binding of glucose-modified albumin is associated with increased monolayer permeability and modulation of cell surface coagulant properties. J. Exp. Med. 170: 1387-1407.

16. Schmidt A-M, Zhang J, Crandall J, et al. (1994) Interaction of advanced glycation endproducts with their endothelial cell receptor leads to enhanced expression of VCAM-1: A mechanism for augmented monocyte-vessel wall interactions in diabetes. F.A.S.E.B. J. 8(Part II): A662. Abstract.

17. Kirstein M, Brett J, Radoff S, Stern D, Vlassara H. (1990) Advanced glycosylation endproducts selectively induce monocyte migration across intact endothelial cell monolayers, and elaboration of growth factors: Role in aging and diabetic vasculopathy. Proc. Natl. Acad. Sci. U.S.A. 87: 9010-9014.

18. Bucala R, Tracey KJ, Cerami A. (1991) Advanced glycosylation products quench nitric oxide and mediate defective endotheliumdependent vasodilatation in experimental diabetes. J. Clin. Invest. 87: 432-438.

Contributed by K. S. Warren on March 16, 1995.
19. Yan S-D, Schmidt A-M, Anderson G, et al. (1994) Enhanced cellular oxidant stress by the interaction of advanced glycation endproducts with their receptors/binding proteins. J. Biol. Chem. 269: 9889-9897.

20. Vlassara H, Fuh $H$, Makita Z, Krüngkrai S, Cerami A, Bucala R. (1992) Exogenous advanced glycosylation endproducts induce complex vascular dysfunction in normal animals; A model for diabetic and aging complications. Proc. Natl. Acad. Sci. U.S.A. 89: 12043-12047.

21. Yang CW, Vlassara $\mathrm{H}$, Peten EP, He CJ, Striker GE, Striker LJ. (1994) Advanced glycosylation endproducts upregulate gene expression found in diabetic glomerular disease. Proc. Natl. Acad. Sci. U.S.A. 91(20): 9436-9440.

22. Vlassara H, Striker LJ, Teichberg S, Fuh H, Li YM, Steffes M. (1994) Advanced glycosylation endproducts induce glomerular sclerosis and albuminuria in normal rats. Proc. Natl. Acad. Sci. U.S.A. 91: 11704-11708.

23. Makita Z, Vlassara H, Cerami A, Bucala R. (1992) Immunochemical detection of advanced glycosylation endproducts in vivo. $J$. Biol. Chem. 267: 5133-5138.

24. Edwards CA, O'Brien Jr WD. (1980) Modified assay for determination of hydroxyproline in a tissue hydrolysate. Clin. Chim. Acta. 104: 161-167.

25. Wing AJ, Brunner FP, Brynger HOA, Jacobs C, Kramer P. (1986) In: Drukker W, Parsons FM, Maher JF (eds). Replacement of Renal Functions by Dialysis. 2nd Ed. Nijhoff, Dordrecht, pp. 850-871.

26. Bucala R, Makita Z, Vega G, et al. (1994) Modification of low density lipoprotein by advanced glycation end products contributes to the dyslipidemia of diabetes and renal insufficiency. Proc. Natl. Acad. Sci. U.S.A. 91: 9441-9445.

27. Vlassara $H$, Brownlee $M$, Manogue KR, Dinarello C, Pasagian A. (1988) Cachectin/TNF and IL- 1 induced by glucose-modified proteins: Role in normal tissue remodeling. Science 240: 1546-1548.

28. Steinberg D, Parthasarathy S, Carew TE, Khoo JC, Witztum JL. (1989) Beyond cholesterol. Modifications of low-density lipoprotein that increase its atherogenicity. $N$. Engl. J. Med. 320: 915-924. 